\title{
Contar con la infancia en situación de riesgo en los procesos de investigación: pasos hacia la coproducción
}

\author{
Ainoa Mateos Inchaurrondoํㅜ Eduard Vaquero Tió2; Aida Urrea Monclús ${ }^{3}$; Belén Parra Ramajo ${ }^{4}$
}

Recibido: 12 de febrero de 2020 / Aceptado: 19 de junio de 2020

Resumen. De acuerdo con la Convención sobre los Derechos del Niño, los y las niñas tienen derecho a participar en todos aquellos aspectos que les afectan. Se reconoce cada vez más la importancia de dar voz y tener en cuenta a la infancia. Sin embargo, esta participación se ve limitada en algunas ocasiones, especialmente en contextos como el sistema de protección a la infancia o en contextos en los que ésta se halla en una situación de riesgo o vulnerabilidad. Por ello, es necesario promover experiencias en las que niños, niñas y adolescentes sean agentes activos de la investigación. Las aportaciones de los principales implicados dan significatividad a los hallazgos y acercan la investigación al foco de estudio, así como a los mismos agentes. Las investigaciones deben incorporar a la infancia y adolescencia no sólo como fuente de información sino como agentes principales. En el presente artículo se muestran algunas de las metodologías de investigación participativas utilizadas, a lo largo de estos últimos años, por el por el grupo de investigación en intervenciones socioeducativas en infancia y juventud (GRISIJ). Los ejemplos sitúan el foco de la investigación en la infancia en riesgo y en su derecho a participar en los procesos de investigación sobre cuestiones que les afectan. Finalmente, se proponen algunas ideas para adquirir mayor compromiso con la participación infantil en estos procesos, especialmente, en los casos de infancia en situación de riesgo o en el sistema de protección.

Palabras clave: Derechos de la infancia; participación infantil; infancia en riesgo; investigación; técnicas.

\section{[pt] Contar com a infância em risco nos processos de pesquisa: passos para a coprodução}

Resumo. De acordo com a Convenção sobre os Direitos da Criança, meninas e meninos têm o direito de participar de todos os aspectos que os afetam. A importância de dar voz e levar em conta a infância é cada vez mais reconhecida. No entanto, essa participação é limitada em algumas ocasiões, especialmente em contextos como o sistema de proteção à criança ou em contextos em que se encontra em situação de risco ou vulnerabilidade. Portanto, é necessário promover experiências nas quais os agentes ativos da investigação sejam as crianças mesmas. As contribuições dos principais interessados dão relevância aos resultados e aproximam a pesquisa do foco do estudo, bem como dos próprios agentes. Os estudos devem incorporar a infância e a adolescência não apenas como fonte de informação, mas como principais agentes. Este artigo mostra algumas das metodologias de pesquisa participativa utilizadas, nos últimos anos, pelo grupo GRISIJ (Grupo de Pesquisa sobre intervenções socioeducativas na infância e juventude).. Os exemplos dos estudos colocam o foco da pesquisa em crianças em risco e em seu direito de participar de processos de pesquisa sobre questões que as afetam. Por fim, propõese algumas ideias para adquirir, do campo científico, maior comprometimento com a participação das crianças nesses processos, principalmente nos casos de crianças em risco ou no sistema de proteção.

Palavras chave: Direitos da criança; participação infantil; infância em risco; pesquisa; técnicas.

\section{[en] Taking into account Childhood at Risk in the Research Processes: Steps towards Coproduction}

Abstract. According to the Convention on the Rights of the Child, children and adolescents have the right to participate in all aspects of life that affect them. The importance of giving a voice and taking into account childhood is increasingly

\footnotetext{
Departamento de Métodos y Diagnóstico en Educación de la Facultad de Educación de la Universidad de Barcelona. E-mail: amateos@ub.edu

2 Universitat de Lleida. Facultad de Educación, Psicología y Trabajo Social. Departamento de Pedagogía.

E-mail: eduardvt@pip.udl.cat

Universitat Autònoma de Barcelona. Facultad de Ciencias de la Educación. Departamento de Pedagogía Sistemática y Social.

E-mail: Aida.Urrea@uab.cat

4 Universitat de Barcelona. Facultad de Educación.

E-mail: belenparra@ub.edu
}

Soc. Infanc. 4, 2020: 87-98 
recognized. However, this participation is limited on some occasions, especially in contexts such as the child welfare system or in contexts where childhood is in a vulnerable situation or at risk. Therefore, it is necessary to promote experiences in which children and adolescents are active agents of the research. Their contribution as stakeholders gives significance to the findings and brings the research closer to the focus of the study, as well as to the agents themselves. Research should incorporate children and adolescents not only as a source of information but as main agents. This article shows some of the participatory methodologies that are used in research, in recent years, by the GRISIJ Group (Research Group for Social and Educational Interventions in Child and Youth). The examples focus on children at risk and on their right to participate in research processes on issues that affect them. Finally, some ideas are proposed to acquire a greater commitment to children's participation in these processes, especially in cases of children at risk or in the child welfare system.

Keywords: Children's rights; child participation; childhood at risk; research; techniques.

Sumario. 1. La infancia en situación de riesgo en el foco de la investigación 2. Enfoques participativos en investigación. 3. Experiencias de investigación con infancia desde una metodología participativa. 4. Orientaciones para la participación infantil en investigación desde la CDN. 5. Conclusiones. 6. Referencias bibliográficas.

Agradecimientos. La realización de este artículo ha sido posible gracias al soporte de la Obra Social la Caixa (FBG309221 y FBG3086), el Ministerio de Ciencia, Innovación y Universidades (RTI2018-099305-B-C21), el Ministerio de Economía y Competitividad (EDU2014-52921-C2-1-R) y el Ministerio de Ciencia e Innovación (EDU2011-00144-C02-01) del Gobierno de España, la Agencia Ejecutiva en el Ámbito Educativo, Audiovisual y Cultural de la Unión Europea (2018-1-FR01KA202-048115), y la Agencia de Gestión de Ayudas Universitarias y de Investigación de la Generalitat de Cataluña (2017SGR905). Los y las autoras quieren agradecer a los niños, niñas y adolescentes su participación en los diferentes proyectos de investigación.

Cómo citar: Mateos, A., Vaquero, E., Urrea, A., \& Parra, B. (2020). Contar con la infancia en situación de riesgo en los procesos de investigación: pasos hacia la coproducción, Sociedad e Infancias, 4, 87-98.

\section{La infancia en situación de riesgo en el foco de la investigación}

Los niños y las niñas tienen derecho a participar en todos aquellos aspectos que afectan a sus vidas. Así lo estipula la Convención sobre los Derechos del Niño (CDN) (ONU, 1989). Este marco internacional ha permitido reconocer la importancia de dar voz a la infancia y potenciar su participación en todos aquellos aspectos que promocionen su desarrollo y bienestar (Tisdall, 2017). Sin embargo, tanto la Comisión Europea (REC(2013)/112/UE) como el Consejo de Europa (CM/REC(2011)12; CM/REC(2012)2) identificaron barreras al respecto y recomendaron que se posibilite el desarrollo de los derechos de participación de los niños, las niñas y los y las adolescentes. En consecuencia, la política actual en materia de protección a la infancia (Ley 26/2015, de 28 de julio, de modificación del sistema de protección a la infancia y a la adolescencia) añade artículos específicos que apuestan por escuchar y reconocer a la infancia como agentes activos en todos aquellos procesos que les incumben. Cada vez son más los estudios que señalan la importancia de dar voz y escuchar las opiniones de la infancia y la adolescencia. Y aún con mayor significación en aquellos casos en los que este colectivo se halla en una situación de riesgo o vulnerabilidad (Dixon, Ward y Blower, 2019), incluyendo los procesos de protección a la infancia, así como, investigaciones sobre la misma (Mitchell et al., 2010; Schnoor, 2013).

Pese a la voluntad política, este derecho no siempre está garantizado en aquellos casos en los que los niños y las niñas se encuentran en el sistema de protección. En este contexto, y transferible a otros, se dan intervenciones profesionales en las que se toman decisiones importantes sobre la vida de los niños y las niñas sin informarles o tener en consideración su opinión. No se trata sólo de dar voz sino de legitimarla y reconocerla (Mateos et al., 2017). En este sentido, la participación en la infancia debe ser entendida no sólo como un derecho, sino como una experiencia personal y colectiva, que posibilita la implicación en proyectos sociales y educativos (Lay-Lisboa et al., 2018). Goodyer (2014) corrobora en su estudio cómo las decisiones dentro del sistema de protección a la infancia no tienen en cuenta ni el punto de vista de los niños, las niñas y los adolescentes, ni tampoco suelen atender a sus necesidades de estar adecuadamente informados sobre las medidas y decisiones que se han tomado con respecto a su situación familiar. Esta falta de participación, de información y de tiempo para procesarla se ha constatado en diversos estudios nacionales e internacionales (Mitchell et al., 2010; Montserrat, 2014; Mateos et al., 2017, 2018). En esta línea, el estudio de Staines y Selwyn (2020) señala que una proporción significativa de niños, niñas y adolescentes de su estudio sintieron que no habían recibido ninguna información o que ésta fue parcial, sobre los motivos que dieron lugar a la separación de su familia biológica. Coincidiendo con estos datos, Balsells, Vaquero y Ciurana (2019) señalan este déficit de información en los niños y las niñas acogidas, y añaden que a éstos les hubiese gustado que el apoyo social recibido hubiera servido como fuente informacional en los momentos iniciales del proceso de acogida.

Para fomentar la participación infantil en el sistema de protección a la infancia, como señalan Cossar, Brandon y Peter (2016) es necesario que se den algunas circunstancias tales como: que el profesional establezca una relación de 
confianza con el niño, niña o adolescente, posibilitando un espacio en el que estos puedan sentirse cómodos para expresarse; que el contacto entre niño, niña o adolescente y el profesional sea frecuente y continuado y que contribuya al establecimiento de una relación de confianza; y por último, evitar que éstos encuentros se conviertan en interrogatorios y momentos cuya finalidad exclusiva sea la de recabar información y/o evaluar. En la misma línea, Cashmore y O'Brien (2001) enuncian seis condiciones para la participación efectiva: la oportunidad y la elección de las forma de participar; el acceso a la información; la posibilidad de profesionales de confianza; la dotación de recursos y apoyo para poder participar; la disponibilidad de procesos y de estructuras apropiadas; $y$, la existencia de procesos y gestión de quejas. A estas condiciones, pueden añadirse otras implicaciones prácticas, que señalan Balsells, Vaquero y Ciurana (2019), para la participación infantil en los procesos de acogimiento: relación sincera y transparente entre profesionales y acogidos; relación próxima y empática; utilización de lenguaje no verbal como estrategia de acercamiento y apoyo; trabajar los sentimientos de culpabilidad; y, la corresponsabilidad del apoyo social.

Coincidiendo con Dixon, Ward y Blower (2019) resulta llamativo que a la infancia y adolescencia tutelada, que es interrogada de forma reiterada, por diferentes profesionales, sobre sus historias y situaciones familiares, se le niegue la voz y el derecho a expresarse cuando se trata de tomar decisiones sobre sus vidas. Si bien se ha tratado de mejorar las formas de intervención profesional en la infancia, abandonando modelos de intervención de carácter asistencial y paternalista, para apostar por un modelo en el que el niño, la niña o adolescente se halle en el foco de la intervención (Balsells, Fuentes-Peláez y Pastor, 2017), la realidad sigue siendo que su voz está silenciada tanto en el sistema de protección como en los estudios e investigaciones sobre la infancia y la adolescencia en situación de riesgo. En esta línea, Dixon, Ward y Blower (2019) señalan que pese a la baja participación infantil existe una reciente transición hacia una mayor participación de los niños en los estudios. De ahí, la importancia de incorporar en las investigaciones enfoques y técnicas que posibiliten una participación real en la infancia.

Como señala Dillon (2018) en los estudios sobre infancia y adolescencia, los niños deben ser colocados en el foco de atención, deben ser los sujetos de la investigación, más que el objeto. Los niños y las niñas son sujetos de derechos y situarlos en el centro de la investigación afecta positivamente a diferentes áreas de su desarrollo: se reafirman como seres con capacidad de comprensión, interpretación, negociación, conciliación y expresión (Lundy y McEvoy, 2012; Rodríguez-Pinto y Albuquerque, 2018; Templeton et al., 2019), y, en consecuencia, se convierten en actores principales de sus vidas (Shier, 2019).

Los avances en la conceptualización de la infancia han supuesto un cambio en el foco y en la posición de ésta en las investigaciones de tipo social, lo que refleja una progresiva incorporación de los derechos de la infancia en diferentes esferas, entre ellas, la investigación (Davidson, 2017; Flewitt et al., 2018). Esto supone la búsqueda del equilibrio de poderes dentro de las investigaciones entre los niños y las niñas y el equipo de investigación de adultos (Shier, 2019). Se trata de ir más allá de métodos participativos, asumiendo los principios de inclusión social y reciprocidad (Davidson, 2017) así como de configurar investigaciones acordes con los derechos de la infancia y que permitan a niños, niñas y adolescentes expresarse, en un espacio propio, donde alguien les escuche y, finalmente, sus ideas y opiniones sean adoptadas (Lundy, 2007). En la línea de las aportaciones de Flewit et al. (2018) y Templeton et al. (2019), existen grandes diferencias entre el papel del adulto interpretando las perspectivas de los niños y niñas y la realidad y perspectivas que aportan estos mismos cuando se les brinda la oportunidad.

Los niños y las niñas pueden aportar contribuciones importantes a la investigación, ya que éstos, son los actores principales. Sin embargo, estudiar sobre la infancia con la infancia supone que la investigación sea algo más compleja (Dillon, 2018), ya que requiere adaptar las técnicas y los enfoques de la investigación a estos actores. Todo ello, configura un reto importante para promover la participación infantil en el diseño de técnicas que recojan los múltiples lenguajes de los más pequeños (Castro, Ezquerra y Argos, 2016).

\section{Enfoques participativos en investigación}

La literatura científica enfatiza el valor de los enfoques participativos en la investigación basándose en dos argumentos principalmente. El primero expone que en las investigaciones participativas el conocimiento producido es "mejor", al facilitar una comprensión más matizada de las situaciones sociales más complejas (Gallagher, 2008), de igual forma, al valorarse el discurso subjetivo de la voz de la infancia, los adultos descubren como ésta ve y experimenta el mundo (Templeton et al., 2019). El segundo argumento es de carácter ético, se concibe que la investigación participativa al dar la voz a los protagonistas, se trasforma en una investigación emancipadora (Palaiologou, 2014; Horgan, 2016)P., \& Fuller, M. (1996.

La investigación participativa engloba una serie de métodos y un conjunto de técnicas con la característica común de involucrar activamente a los sujetos de la investigación, lo que implica un enfoque colaborativo y no jerárquico (Gallagher, 2008; Bird et al., 2020). De esta forma, el investigador cambia de rol y se convierte en un facilitador. En consecuencia, tiene la función de ayudar a los participantes a producir conocimiento sobre ellos mismos (Bouma et al., 2018).

Para afrontar el reto de hacer más participativas las investigaciones en las que niños, niñas o adolescentes sean el foco de estudio, resulta básico buscar enfoques, metodologías y técnicas de investigación que posibiliten a la infancia y a la adolescencia oportunidades reales de participación. El modelo de participación de Lundy (2007) conceptualiza 
el Artículo 12 de la Convención sobre los Derechos del Niño y propone 4 elementos a tener en cuenta: (1) espacio, se deben garantizar oportunidades seguras e inclusivas a la infancia para formar y expresar sus opiniones; (2) voz, debe facilitarse a la infancia que exprese sus opiniones; (3) audiencia, sus opiniones deben ser escuchadas; y (4) influencia, las opiniones deben aplicarse, si son apropiadas. En este sentido, hay otros muchos modelos de participación (Hart, 1992; Shier, 2001, 2019; Lansdown, 2011; Bouma et al., 2018) que contemplan estos elementos.

A continuación, presentamos cuatro niveles de investigación, definidos por Dixon, Ward y Blower (2019), que pueden brindar a la infancia una oportunidad de participación no sólo en la investigación sino también en los servicios que la atiende. Estos enfoques son: participación, consulta, investigación entre pares y co-producción.

\section{Participación}

Dentro de este nivel se incluyen el uso de pruebas de investigación dirigidas a informar sobre la práctica profesional y que los servicios conozcan y respondan a las voces de las experiencias de los niños, las niñas y adolescentes. El desafío que supone este enfoque, según los autores citados, es garantizar que la voz de la infancia no quede diluida o distorsionada por la interpretación del adulto investigador. Para minimizar este sesgo se introducen y diseñan métodos que tratan de colocar a la infancia en el centro del proceso de investigación.

\section{Consulta}

La consulta amplía la participación tradicional de los niños, niñas y adolescentes como sujetos de la investigación, ofreciéndoles un rol de asesoría para el impacto directo de los procesos de estudio. Este acto consultivo se puede dar mediante entrevistas, grupos focales y/o encuestas.

\section{Investigación por pares}

Este enfoque, integrado en métodos participativos, permite a los niños, las niñas y los adolescentes participantes adoptar, dentro del grupo temático, un rol de investigador. Desde este enfoque, estos se incluyen en el proceso de investigación a diferentes niveles: asesoría sobre las premisas de investigación, instrumentos de recolección de datos, análisis de los datos, actividades de difusión, etc.

\section{Coproducción}

En este enfoque, los participantes en la investigación, es decir las personas objeto de estudio y los investigadores, diseñan y desarrollan conjuntamente el trabajo. La coproducción garantiza que los niños, las niñas y/o los adolescentes sean agentes activos en aquellos productos que se generan para abordar sus necesidades. Se trata de un estilo de trabajo colaborativo en la que los investigadores adultos y los niños y las niñas se hallan en un mismo nivel de importancia. Unos son expertos académicos o técnicos y los otros expertos como personas que viven esa situación o experiencia.

Teniendo en cuenta todos los elementos aportados hasta ahora, y de acuerdo con Davidson (2017), podemos señalar que uno de los rasgos que caracteriza a una investigación participativa con infancia es la preocupación epistemológica por la mutualidad, la igualdad de los actores y el empoderamiento de las niñas, niños y adolescentes con el objetivo de democratizar los procesos de producción de conocimiento.

En resumen, uno de los debates actuales en la literatura científica sobre la infancia en los procesos de investigación es el alcance real de la participación de las niñas, los niños y los adolescentes en los estudios. Gallagher (2008) señala que si bien los enfoques participativos son bastante comunes en el proceso de la recogida de datos, la participación de estos actores en las fases de planificación, análisis y difusión suele estar más limitada. Es por ello que se debería avanzar hacia un enfoque participativo más pleno y explorar como involucrar a los niños, las niñas y los adolescentes en el diseño de los estudios, en la construcción de los instrumentos, en la interpretación de los resultados y el proceso de escritura del informe final.

Sin duda, la investigación participativa puede tener diferentes diseños y utilizar diversos métodos y técnicas como mostramos a continuación.

\section{Experiencias de investigación con la infancia desde una metodología participativa}

Como se ha expuesto en líneas anteriores, cada vez son más las investigaciones que tratan de incluir en diferentes ámbitos de estudio, la voz de la infancia y la adolescencia en sus investigaciones (Lundy y McEvoy, 2012; Coiduras et al., 2016; Dixon, Ward y Blower, 2019; Shier, 2019; Templeton et al., 2019). La mayoría de estas investigaciones se sitúan en enfoques metodológicos cualitativos, y han venido empleando técnicas de recogida de datos como la entrevista o los grupos de discusión. Estas técnicas permiten recoger la voz directamente de los niños y niñas participantes de manera que puedan expresarse libremente. 
No obstante, tradicionalmente estas técnicas se basan en una comunicación verbal, es decir, en la respuesta a preguntas definidas por los investigadores. Este hecho proporciona, al fin y al cabo, una información limitada. Por un lado, los participantes pueden sentirse cohibidos para responder ciertas cuestiones delante de personas que la mayoría de las veces no conocen y con quienes no tienen confianza. Por otro, las preguntas acostumbran a ser complejas y poco ajustadas en vocabulario o nivel de abstracción a las características de los participantes, lo que dificulta su comprensión, respuesta y recogida de información (Balsells, Fuentes-Peláez y Pastor, 2017). Estas limitaciones añaden un sesgo, no solo en las informaciones que se recogen y se analizan posteriormente, sino que, además, suponen un sesgo en las conclusiones de los estudios, en las orientaciones profesionales que pueden transformar la práctica, así como en la mejora de la calidad de vida de la infancia y la adolescencia.

Phoenix, Orr y Griffin (2017) sugieren que, para contrarrestar estas limitaciones, pueden usarse, de forma complementaria a las técnicas tradicionales de recogida de datos, otros recursos y estrategias metodológicas que favorezcan la reflexión y el dialogo de los participantes. Weber y Mitchell (1996) también apuntan hacia el arte de dibujar como una estrategia complementaria a los grupos de discusión o entrevistas, ya que posibilita a los niños y las niñas comunicar sus experiencias en sus propios términos, y los animan a participar, mediante el dibujo. Este recurso les invita a habar desde la metáfora y potencia la comunicación desde un lenguaje más próximo a la infancia.

En este sentido, Morgan (2017) argumenta que los procedimientos de recopilación de datos deben evolucionar, para construir, sobre lo que ya sabemos, nuevos enfoques, diseños y estrategias de recogida de datos que permitan dar voz a los participantes y crear espacios más facilitadores para la interacción, comunicación e intercambio de información.

Estas innovaciones son especialmente interesantes si están dirigidas a fomentar la participación de niños y niñas (Berson, Berson, y Gray, 2019). Por ello, se presentan a continuación tres experiencias de investigación del grupo GRISIJ realizadas mediante estrategias de recogida de datos basadas en las entrevistas y grupos de discusión, a las cuales se añadieron algunas innovaciones a la técnica tradicional para promover y facilitar la participación de los niños y las niñas.

Cabe destacar, por la importancia que esto supone en términos de ética en la investigación, que estas experiencias se guiaron por el máximo rigor y ética científica. En todos los casos se garantizaron los derechos a la protección de datos y la confidencialidad de estos, así como el derecho de los niños y las niñas a participar de forma voluntaria en la investigación, respetando sus silencios cuando estos se produjeron.

Las experiencias que se presentan han sido seleccionadas teniendo en cuenta la competitividad de los proyectos, el impacto y transferencia del conocimiento que éstos han supuesto y la diversidad de técnicas para promover la participación infantil. El orden en que se presentan está relacionado con la reflexión y los aprendizajes realizados a partir de la propia práctica de investigación empleando metodologías participativas para dar voz a la infancia. El proceso de evaluación-reflexión realizado por el equipo de investigación tras cada una de las distintas experiencias, ha permitido aumentar el nivel de participación de los niños y niñas en las sucesivas experiencias, con el reto de alcanzar la coproducción.

\section{Experiencia 1. Caminar en familia}

"Caminar en familia" es un programa socioeducativo de competencias parentales durante el acogimiento y la reunificación familiar (Balsells et al., 2015) desarrollado, implementado y evaluado en el marco de tres proyectos I+D (EDU2011-00144-C02-01, EDU2014-52921-C2-1-R y RTI2018-099305-B-C21). El programa tiene como finalidad el apoyo a las competencias parentales específicas que las familias tienen que desarrollar en un proceso de acogida y de reunificación familiar. Su principal objetivo es promocionar la aceptación y la implicación de la medida de protección para facilitar el regreso de los hijos e hijas lo más pronto posible al hogar y afianzar la reunificación una vez están en casa.

El diseño, implementación y evaluación de este recurso socioeducativo se desarrolló escuchando la voz de niños y niñas del sistema de protección a través de entrevistas y grupos de discusión. Por un lado, las entrevistas permiten recoger experiencias, vivencias y hechos cotidianos poniendo énfasis en las narraciones de los entrevistados en su contexto natural (Hernández, 2014). Por otro lado, los grupos de discusión permiten recoger información mediante la confrontación de ideas de los y las participantes del grupo artificial formado (Lara y Ballesteros, 2007). Estas técnicas de recogida de información se adaptaron para favorecer la voz, la escucha y la influencia de los niños y de las niñas participantes ( $17 \%$ con edades entre 6 y 11 años; 56\% con edades entre $12-17$ años; y $27 \%$ mayores de 18 años) en la investigación.

En primer lugar, se diseñó un guión de preguntas que orientara la recogida de información teniendo en cuenta que el vocabulario empleado fuera afín y cercano al vocabulario infantil. Se adaptó el formato en función de la edad y la madurez de los y las participantes tanto de los grupos de discusión con niños y niñas como en la entrevista para adolescentes. En este sentido se emplearon expresiones más coloquiales. En segundo lugar, la adaptación de las preguntas que conformaron el guión se ajustó también a los tiempos, haciéndolos lo más precisos posible, para facilitar así su comprensión. Un ejemplo de ambas es el siguiente: en lugar de preguntar ¿qué información recibisteis cuando se inició el proceso de acogimiento?, se ajustó el vocabulario y el momento a ¿qué te dijeron antes de irte de casa? 
En tercer lugar, también se modificaron algunos aspectos procedimentales de las técnicas de recogida de datos empleadas: entrevistas y grupos de discusión. Por un lado, se realizaron las entrevistas en espacios conocidos por los niños y las niñas participantes. Por otro, antes de iniciar las preguntas de contenido en los grupos de discusión, se realizaron pequeñas dinámicas de presentación o de cohesión grupal con la finalidad de acercarse al grupo de niños y niñas participantes, distender el ambiente y facilitar la comunicación e intercambio de experiencias.

A pesar de estas adaptaciones, y de los esfuerzos por parte de los y las investigadoras para facilitar la participación infantil, en algunos grupos de discusión ciertos participantes se mostraron cohibidos y con reticencias para comunicar y expresar verbalmente sus ideas y aportaciones. A pesar de recoger información valiosa, este hecho nos condujo al reto de indagar en nuevas técnicas que permitieran garantizar que la voz de todos los niños y las niñas participantes pudiera ser escuchada y considerada. De ahí, que en posteriores investigaciones y evaluaciones se optara por buscar técnicas multilenguaje que posibilitaran ser más inclusivos con la diversidad de participantes y recoger así de una forma más cercana las aportaciones de los niños y las niñas.

\section{Experiencia 2. Aprender juntos, crecer en familia}

"Aprender juntos, crecer en familia" es un programa socioeducativo cuya finalidad es contribuir al bienestar familiar (FBG309221 y FBG3086). El objetivo general del programa es promocionar el desarrollo y la convivencia familiar mediante el fomento de las relaciones positivas entre padres, madres e hijos e hijas ( 6 a 12 años) de acuerdo con el ejercicio de una parentalidad positiva. El programa responde a una visión holística de un proceso de desarrollo de la convivencia familiar desde una triple dimensión: dimensión emocional, que pretende ayudar a manejar les emociones; dimensión comportamental, por medio del desarrollo de habilidades que permiten afrontar de forma competente las situaciones; $y$, dimensión cognitiva, que facilite una mayor comprensión del proceso de convivencia familiar (Amorós et al., 2012, 2013, 2015, 2016; Rodrigo et al., 2013).

En las diferentes evaluaciones (2011-2015) del proceso de implementación e impacto del programa (Amorós et al., 2016) se emplearon, entre otras, técnicas de recogida de datos cualitativas, como los grupos de discusión con niños y niñas que participaron en el programa. Sin embargo, esta técnica tradicional y común para recabar información con la infancia, genera ciertas limitaciones que el grupo de investigación ha podido constatar a lo largo de su trayectoria investigadora con infancia y adolescencia: los niños y las niñas, especialmente los más pequeños, muestran dificultades en su narrativa y tienden a dar respuestas sencillas, estereotipadas y miméticas. Todo ello llevó al equipo investigador a plantearse la necesidad de hallar técnicas de recogida de información alternativas para poder captar la voz directa de los niños y las niñas.

De esta manera, teniendo en cuenta los precedentes en este proyecto así como en investigaciones anteriores como la citada en la experiencia 1, se sustituyó el uso de la técnica de los grupos de discusión con los niños y las niñas por entrevistas grupales semiestructuradas mediante el uso de la metodología Arts Based Research (ABR) (Hernández, 2008; Jackson, Deafenbaugh y Miller, 2018). Esta metodología permite expresar vivencias personales de manera más libre y personalizada. Así mismo, el dibujo como estrategia de comunicación, les permite expresarse sin una interpelación excesiva del adulto, lo que ayuda a transmitir estados emocionales y experiencias personales difíciles de contar verbalmente (Weber y Mitchell, 1996).

Las entrevistas grupales se llevaron a cabo en grupos de entre tres y cinco niños o niñas (6 a 12 años), ya que la literatura científica avala que la ratio del grupo debe situarse en torno a los tres participantes, especialmente, cuando queremos preguntar sobre los conocimientos o el grado de satisfacción respecto a algo (Mauthner, 1997; Leitch y Mitchell, 2007).

Una vez creado un ambiente y clima agradable, cómodo y de proximidad para el desarrollo de la entrevista, se introdujeron los objetivos y el procedimiento a seguir con un lenguaje ajustado a la edad y desarrollo madurativo de los y las participantes. En primer lugar, se les pidió realizar un dibujo en base a un enunciado (Figura 1). Un ejemplo fue: De todo lo que has aprendido, dibuja lo que más te ha gustado. De esta manera, se recogió información sobre los aprendizajes más significativos, así como, la propia vivencia de su participación en el programa.

En segundo lugar, se comentó el dibujo con el niño o niña indagando en aquello que quería expresar, pero sin hacer una interpretación desde el punto de vista del investigador. Así, el instrumento de recogida de datos ayudó al investigador a plantear preguntas que facilitaran la expresión verbal de los niños y las niñas como, por ejemplo: ¿Si tuvieseis que explicar tu dibujo a otro niño, qué le diríais? Las respuestas de los niños y las niñas fueron grabadas y tratadas como la locución de respuesta a una entrevista tradicional. 


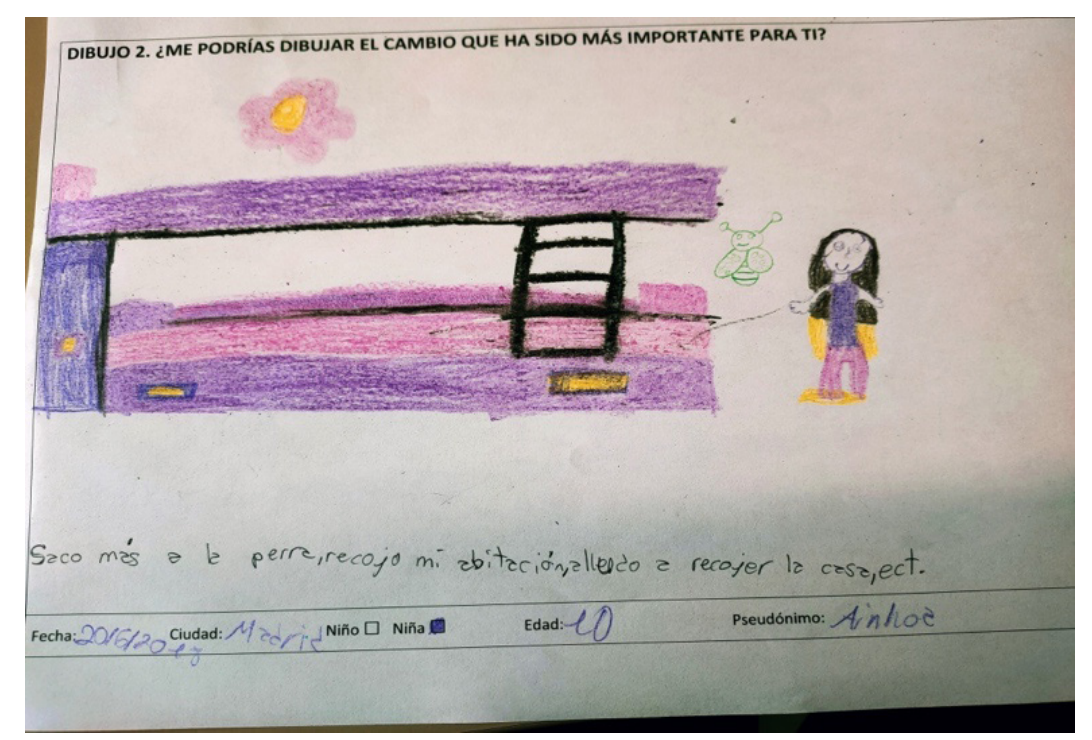

Figura 1. Ejemplo de dibujo realizado por una niña de 10 años, Madrid (2018).

Esta metodología permitió que niños y niñas pudieran expresar sus vivencias personales de manera más libre y personalizada, gracias a que el dibujo, como estrategia de expresión, permite crear una situación en la que el niño o la niña comunican sin interpretación de la persona adulta investigadora.

\section{Experiencia 3. Grow In Family Today (GIFT)}

El proyecto GIFT (2018-1-FR01-KA202-048115) es un proyecto de investigación europeo donde están implicados cuatro países: España, Francia, Italia y Rumanía. La investigación tiene como finalidad promover el desarrollo de relaciones positivas entre padres, madres, niños y niñas dentro del entorno familiar, tanto en sus dimensiones emocionales, relacionales como educativas. Entre sus objetivos está conocer la evolución y la diversidad de las nuevas formas de familia, contribuir a mejorar las prácticas profesionales para apoyar el ejercicio de la parentalidad y fomentar que los niños y las niñas crezcan en un entorno favorable.

Desde un enfoque cualitativo, esta investigación incluyó técnicas de recogida de información mediante entrevistas y grupos de discusión. Con la finalidad de armonizar la recogida de información en países culturalmente distintos y de fomentar la participación de todos los informantes, se diseñó una estrategia de recogida de datos que, si bien partía del grupo de discusión, incluía el uso de imágenes del juego de mesa Dixit (Figura 2). Este juego de mesa, creado en 2008 por Jean-Louis Roubira, tiene como objetivo adivinar una carta a partir de pistas que se dan en forma de frase o narración breve. El uso de estas imágenes ha sido sugerido como "una fuente adicional de inspiración" por parte de algunos autores cuando son usadas en combinación con métodos y técnicas de recogida de datos tradicionales (Wetzel, Rodden y Benford, 2017: 206).

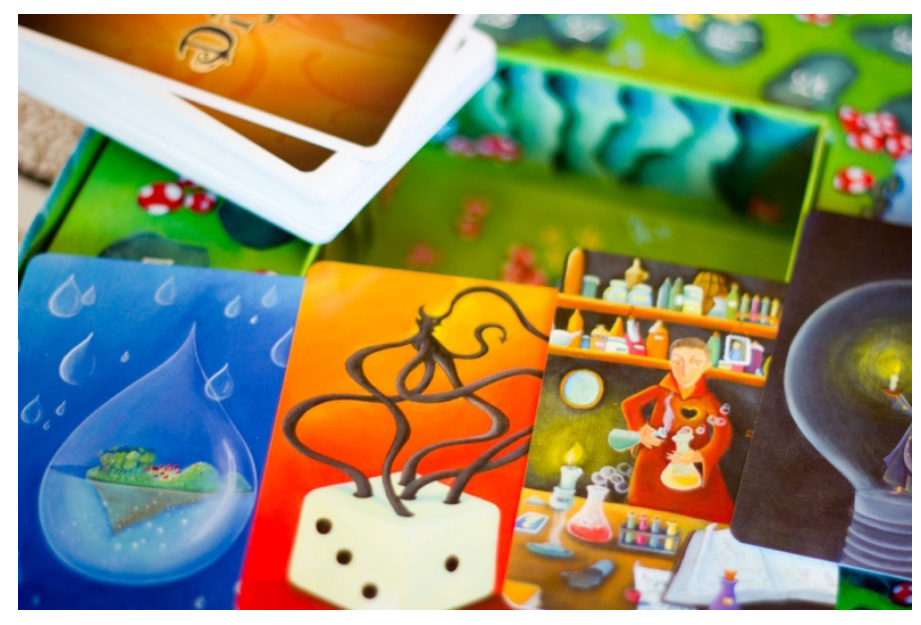

Figura 2. Cartas del juego Dixit (Santos, 2014).

En este proyecto, la dinámica se dividió en dos fases: la fase de narración y la fase de reflexión. En la fase de narración, el investigador y dinamizador del grupo de discusión expuso el tema a tratar, informando del título y una breve descripción del mismo sin realizar preguntas. Al mismo tiempo, otro investigador colaborador mezcló las 
cartas y las dispuso encima de una mesa boca arriba, esparciéndolas aleatoriamente para que todos los participantes pudieran observar el mayor número de imágenes posible. Acto seguido, se pidió a los y las adolescentes (10-17 años) que observasen las cartas y tratasen de escoger alguna que les sugiriera alguna idea sobre el tema expuesto, dejando un minuto como máximo para que todos los participantes hicieran su elección. Una vez terminado el tiempo se inició la segunda fase de reflexión.

En la fase de reflexión, el investigador informó de que las cartas son un pretexto para generar debate y reflexión en los participantes y que no era necesario que ellos explicasen los motivos de por qué habían escogido cualquiera de las cartas, o que compartieran qué les había generado a nivel emocional o introspectivo en sí mismos. Tras ello el dinamizador planteó a los adolescentes las preguntas del guión del grupo de discusión sobre el tema tratado.

Una vez terminadas las preguntas sobre un tema, se volvió a plantear un nuevo ciclo de narración-reflexión para los siguientes temas. Para ello, los participantes devolvieron las cartas, se colocaron encima de la mesa boca arriba, se mezclaron brevemente y se plantearon los nuevos temas y preguntas del grupo de discusión.

El uso de las imágenes que aparecen en las cartas del juego de mesa Dixit permitió la dinamización de los grupos de discusión, facilitando la reflexión, el debate e intercambio de ideas, apelando a un pensamiento creativo y a un lenguaje más próximo a la infancia, como es el visual. En esta experiencia, el equipo investigador apostó por integrar técnicas que venía utilizando en estudios anteriores, como por ejemplo la entrevista y el grupo de discusión, en combinación con un soporte visual como las cartas del juego Dixit. Esta metodología supone una variante en el uso de técnicas de recogida de información multilenguaje con respecto a la anterior experiencia. En la experiencia 2, se genera el discurso a partir de una creación del participante que sirve de pretexto para motivar el discurso. En la experiencia 3, las imágenes de las cartas son fuente de inspiración para la elaboración del discurso de los y las participantes. En ambos casos la finalidad es la misma: ayudar a emerger la voz de los y las participantes sin interpretación del equipo de investigación.

Son diversos los autores que avalan la necesidad de adoptar la participación desde diferentes dimensiones (Susinos y Ceballos, 2012; Coiduras et al., 2016). No solamente la voz es participación sino que se trata de valorar diferentes formatos que permitan la expresión de niños, niñas y adolescentes. Así es como Coiduras et al. (2016) plantean 5 tipos de formato: oral, textual o escrito, visual o plástico, enactivo o de acción y el digital o multilenguaje. En las propuestas presentadas el enfoque participativo ha pasado de un nivel de participación y escucha oral a un nivel de consulta añadiendo diferentes formatos de consulta, no solo el oral sino incorporando el nivel escrito, visual y enactivo.

\section{Orientaciones para la participación infantil en investigación desde la CDN}

Las distintas experiencias expuestas hasta el momento ponen de manifiesto algunas de las posibilidades para recoger la voz de la infancia y la adolescencia en los procesos de investigación. A su vez, permiten visibilizar cómo incorporar los principios de la Convención sobre los Derechos del Niño en ellos: (1) no discriminación, (2) interés superior del niño, (3) supervivencia y desarrollo, y (4) participación. Así pues, las técnicas e instrumentos de investigación empleadas por el grupo han ido evolucionando y han sido adaptadas para ajustarse a lenguajes más inclusivos y próximos a la infancia, adoptando el principio de inclusión y no discriminación de la CDN.

Esta evolución ha supuesto dos notables avances y beneficios para las investigaciones descritas. Por un lado, escuchar y recoger la voz de niños, niñas y adolescentes permite identificar necesidades particulares y diferenciales relacionadas con su bienestar y desarrollo. Necesidades que son distintas a las que tienen sus padres, madres u otros familiares, así como las que perciben los profesionales que pueden estar implicados. La diferencia entre el punto de vista de la infancia y la adolescencia, y el punto de vista de los agentes implicados a su alrededor, supone reconocer a niños y adolescentes como informantes fundamentales sobre todos los temas que les conciernen, independientemente de cuáles sean estos. Eso nos permite dar cumplimiento a otro de los principios de la CDN, el de libertad de expresión y participación.

Por otro lado, y como consecuencia, el cambio de enfoque está ayudando a superar el estigma que, sin pretenderlo, algunas investigaciones han supuesto cuando únicamente recogían información de niños, niñas y adolescentes vinculados con situaciones de riesgo o vulnerabilidad. Las investigaciones se centran en cuestiones o fenómenos sobre los cuales hay una mayor sensibilidad, urgencia o necesidad de proponer cambios para la mejora del bienestar y calidad de vida de la infancia y la adolescencia. Este hecho redunda en que, en ocasiones, sean los niños y adolescentes en situaciones de riesgo quienes únicamente terminen siendo el foco de investigación, es decir los únicos preguntados o interrogados.

Más allá de estos beneficios, la evolución en las estrategias metodológicas no debe limitarse a facilitar la participación de niños, niñas y adolescentes, reconociéndolos solo como sujetos con un rol meramente participativo y consultivo, sino que se trata de que tanto las investigaciones como los agentes implicados velen por el mayor interés de la infancia en todo aquello que les incumbe, como prescribe la Convención.

De acuerdo con los cuatro niveles definidos por Dixon, Ward y Blower (2019), los procesos de investigación tienen el reto de avanzar hacia niveles que impliquen una intervención más paritaria entre investigadores y participantes, así como de implicar a la infancia y a la adolescencia en todo el proceso investigador. Estos retos ponen de 
manifiesto, por un lado, la necesidad de seguir avanzando y apostar por niveles aún más elevados de participación para poder pasar de la consulta a la investigación por pares y, finalmente, a la coproducción. Y, por otro lado, la necesidad de reconocer el camino que aún queda por recorrer para una participación significativa de la infancia y la adolescencia en los procesos de investigación, que permita cumplir con el principio de interés superior del niño.

Los procesos de investigación pueden y deben implicar la participación de niños, niñas y adolescentes desde un rol activo, considerando dicha participación en todas las etapas del proceso investigador. En esta línea, distintos autores constatan que la participación en la infancia puede realizarse desde distintos niveles y presentan distintos modelos (Hart, 1992; Shier, 2001; Lansdown, 2011) y herramientas (Lansdown y O'Kane, 2014; Shier, 2019) para comprender, repensar y evaluar su grado de participación. Lansdown (2011), por ejemplo, establece, tres niveles de participación esenciales: (1) Consulta, cuando los adultos preguntan a niños, niñas y adolescentes por sus puntos de vista, no involucrándoles más allá; (2) Colaboración, cuando adultos y niños trabajan juntos, comparten roles y responsabilidades en la planificación y realización de una actividad; y (3) Pro-activismo, cuando las actividades son iniciadas, organizadas o dirigidas por niños, niñas y adolescentes. Shier (2019) va más allá y establece una matriz como herramienta práctica para evaluar y repensar la implicación de la infancia y la adolescencia en el proceso investigador. Esta incluye desde el momento de pensar el problema de investigación hasta el diseño de la propuesta o la evaluación de la misma y los relaciona con cuándo, cómo y quién debería estar implicado en cada fase recoge y los niveles de Lansdown, añadiéndole un cuarto nivel donde refleja la no implicación de la infancia.

A pesar de reconocer y tener en cuenta el derecho de los niños a participar en todas las fases de la investigación, existen algunas limitaciones. Y es que a veces es complicado alcanzar el nivel de coproducción expuesto por Dixon (2019) o el nivel de pro-activismo expuesto por Lansdown (2011) en todos los momentos de un proceso de investigación. En este sentido, es difícil alcanzar niveles máximos de participación de niños, niñas y adolescentes en todas las fases de un proyecto de investigación, pero hay que tender hacia la consciencia de que son participantes activos todos aquellos a quienes se debe la investigación. Del mismo modo, "no todos los proyectos de investigación se ejecutan a través de una serie ordenada de etapas" (Shier, 2019: 12). Algunos enfoques de investigación, como la investigación-acción, el Arts Based Research ( $\mathrm{ABR}$ ) o el Design Based Research (DBR), entre otros, tienen diferentes lógicas y procedimientos.

Por todo ello, se presenta a continuación un decálogo de orientaciones con la finalidad de incorporar y promover la participación activa de la infancia y de la adolescencia en los procesos de investigación que les conciernen, en aras del cumplimiento de los principios de la Convención sobre los Derechos del Niño (CDN):

1. Considerar la opinión de los niños, niñas y adolescentes en la selección y priorización de los temas y preguntas de investigación.

2. Reconocerles como informantes clave sobre todos los temas que les conciernen, no solo en aquellos en donde son el foco de la investigación.

3. Legitimar y tener en cuenta sus opiniones como datos válidos, fiables y útiles para la generación de conocimiento, la investigación por pares y la coproducción.

4. Seleccionar instrumentos que permitan recoger directamente sus opiniones, especialmente mediante metodologías cualitativas.

5. Diseñar estrategias de recogida de datos que permitan diversificar las oportunidades de comunicación, basadas en la integración de juegos, dibujos o fotografías, entre otros.

6. Adaptar el lenguaje y el vocabulario a sus necesidades específicas.

7. Delegar, acompañar y guiar a los niños, niñas y adolescentes para que puedan realizar tareas de investigación, especialmente entre iguales.

8. Tenerles en cuenta en los procesos de validación de instrumentos, como unos expertos más a los que poder consultar.

9. Implicarles en los materiales que las investigaciones produzcan, siendo generadores de contenido y/o revisores de los materiales.

10. Considerar a los niños, niñas y adolescentes como promotores y agentes de difusión de los materiales generados.

\section{Conclusiones}

Desde la aprobación por las Naciones Unidas de la Convención sobre los Derechos del Niño (CDN), de 20 de noviembre de 1989, la sensibilidad social hacia la población infantil y adolescente ha ido evolucionando de manera diversa, pero muy notoriamente hacia el rol activo de ésta y su participación en todo aquello que les afecta.

En este artículo se ha puesto de manifiesto la importancia de considerar no sólo el derecho de los niños y adolescentes a participar, sino, de cuestionar cómo estos participan en los procesos de investigación sobre temas que les conciernen como, por ejemplo, en las investigaciones en materia de infancia.

Un elemento importante que se desprende de la literatura científica en el ámbito de la participación infantil es la necesidad de tomar decisiones en las investigaciones en los aspectos relativos a qué se investiga, cómo y con quién. 
Esto alude a la responsabilidad de los equipos investigadores de concienciarse sobre una participación real de la infancia, especialmente, de aquella que se halla en situación de riesgo o vulnerabilidad.

Cada vez son más los estudios que abogan por esta realidad, sin embargo, las investigaciones con y sobre infancia siguen hallándose en unos niveles iniciales, situando los estudios en enfoques de participación y/o consulta. Esto se debe, en parte, a la dificultad de romper con los métodos tradicionales de recogida de información, que no siempre se ajustan a los múltiples lenguajes que se precisan para dar la oportunidad real de participación a la infancia y a la adolescencia. Dar voz a la infancia no se resume en recoger sus ideas o aportaciones, sino que requiere brindar espacios reales de participación, procurar que su voz sea escuchada y que éstas sean incorporadas en las prácticas y en las políticas que se generan en materia de infancia y adolescencia.

Otro elemento a destacar se halla en la importancia de triangular la información en las investigaciones sobre infancia y adolescencia. En muchas ocasiones, los adultos han sido los interlocutores o los agentes informadores en esta materia (como padres, como madres o como profesionales) quedando las aportaciones o las percepciones de los niños en un segundo plano o, incluso, silenciadas. Desde este prisma, sigue siendo el adulto (agente informador) quien determina las necesidades en la infancia desde su propia percepción. Una investigación con la infancia y no sobre la infancia supone apostar por una participación real y brindara los niños y niñas el espacio, el tiempo y el formato idóneo para que expresen sus opiniones; ejercer de audiencia para tener en cuenta sus propuestas e incorporarlas en la triangulación de informaciones de los diferentes agentes que intervienen en el proceso; y, por último, permitir la trasferencia de los resultados de sus aportaciones a la sociedad y a las políticas para que se hagan realidad. Esta transferencia es la que, realmente, brindará oportunidades de participación en las diferentes esferas del desarrollo de los niños, las niñas y los adolescentes.

Para finalizar, todas estas cuestiones desarrolladas llevan a plantearnos retos en la investigación. Nos invitan a pasar de enfoques participativos o de consulta a enfoques más significativos de investigación por pares y/o de coproducción. Todo ello, teniendo presente las limitaciones que supone la participación infantil, como, por ejemplo, la necesidad de adoptar múltiples lenguajes en las técnicas de recogida de información y respetar las cuestiones de privacidad, accesibilidad e información. Esto supone, no sólo garantizar el derecho de los niños y las niñas a que sus datos sean protegidos, sino también, respetar sus silencios y proteger su derecho a participar de forma voluntaria en las investigaciones. Esta participación no debe estar nunca fuera de los rigores éticos de la investigación, por lo que se requiere solicitar el consentimiento o el asentimiento (dependiendo de la edad) para participar en las investigaciones. La Convención sobre los Derechos del Niño, recoge tanto el derecho a la libertad de expresión como el derecho a la privacidad, está en manos de los y las investigadoras velar por que se cumplan estos derechos y se tenga en cuenta en todo momento el interés superior del niño, niña o adolescente.

\section{Referencias bibliográficas}

Amorós, P. et al. (2012). Programa 'Aprender juntos, Crecer en familia'. Barcelona: Obra Social 'La Caixa'.

Amorós, P. et al. (2013). 'Implementation and Evaluation of the "Learning Together, Growing In Family Programme": The Impact On The Families'. Revista de cercetare si interventie sociala, 42, pp. 120-144.

Amorós, P. et al. (2015). Aprender juntos, crecer en familia. Un enfoque integral. En M.J. Rodrigo López et al., Manual práctico de parentalidad positiva (pp. 111-130). Madrid: Síntesis.

Amorós, P. et al. (2016). Learning together, growing with family: The implementationand evaluation of a family support programme'. Psychosocial Intervention, 25, pp. 87-93. https://doi.org/10.1016/j.psi.2016.02.002

Balsells, M. À. et al. (2015). Caminar en familia: Programa de competencias parentales durante el acogimiento y la reunificación familiar. Madrid: Ministerio de Sanidad, Servicios Sociales e Igualdad. Centro de Publicaciones (en línea). http://www. msssi.gob.es/ssi/familiasInfancia/ayudas/docs2013-14/docs2016/CaminarenFamilia.pdf.

Balsells, M. À., Fuentes-Peláez, N., Pastor, C. (2017). Listening to the voices of children in decision-making: A challenge for the child protection system in Spain. Children and Youth Services Review. Elsevier, 79, 418-425. doi: 10.1016/j. childyouth.2017.06.055. https://doi.org/10.1016/j.childyouth.2017.06.055

Balsells, M. À., Vaquero, E., Ciurana, A. (2019). El apoyo social durante el acogimiento: su relevancia para el bienestar de los niños y las niñas en situación de protección. Sociedad e Infancias, 3, 115-132. https://doi.org/10.5209/soci.63403

Berson, I. R., Berson, M. J., Gray, C. (2019). Participatory Methodologies to Elevate Children's Voice and Agency. Research i. Charlotte, NC: Information Age Publishing.

Bird, D. et al. (2020). We are here. A Child Participation Toolbox. Belgium: Eurochild \& Learning for Well-being Foundation.

Bouma, H. et al. (2018). Meaningful participation for children in the Dutch child protection system: A critical analysis of relevant provisions in policy documents. Child Abuse and Neglect, 82(79), 279-292. doi: https://doi.org/10.1016/j.chiabu.2018.02.016.

Cashmore, J., O’Brien, A. (2001). Facilitating participation of children and young people in care. Children Australia. Cambridge University Press, 26(4), 10-15. doi: 10.1017/S1035077200010427.

Castro, A., Ezquerra, P., Argos, J. (2016). Procesos de escucha y participación de los niños en el marco de la educación infantil: Una revisión de la investigación. Educacion XXI, 19(2), 105-126. http://doi: 10.5944/educXX1.14271.

Coiduras, J. et al. (2016). La participación del alumnado en la vida del centro: una aproximación desde la comunidad educativa. Revista Complutense de Educación, 27, 437-456. doi: 10.5209/rev_RCED.2016.v27.n2.46353. 
Comisión Europea (2013). Recomendación de la Comisión de 20 de febrero de 2013. Invertir en la infancia: romper el ciclo de las desventajas (2013/112/UE).

Cossar, J., Brandon, M., Peter, J. (2016). "You've got to trust her and she's got to trust you”: children's views on participation in the child protection system. Child \& Family Social Work, 21(1), 03-112. doi: https://doi.org/10.1111/cfs.12115.

Council of Europe (2011). Recommendation CM/REC (2011)12 of the Committee of Ministers to member states on children's rights and social services friendly to children and families. Europe (en línea). https://www.minori.it/sites/default/files/rec_ cm-rec_2011_12.pdf.

Council of Europe (2012). CM/Rec(2012)2 of the Committee of Ministers to member States on the participation of children and young people under the age of 18 . Europe.

Davidson, E. (2017). Saying it like it is? Power, participation and research involving young people. Social Inclusion, 5(3), 228239. doi: 10.17645/si.v5i3.967.

Dillon, J. (2018). Revolutionizin Participation in Child Protection Proceedings. Liverpool John Moores University for the degree of Doctor of Philosophy. https://doi: 10.13140/RG.2.2.26995.78884.

Dixon, J., Ward, J., Blower, S. (2019). "They sat and actually listened to what we think about the care system": the use of participation, consultation, peer research and co-production to raise the voices of young people in and leaving care in England. Child Care in Practice, 25(1), 6-21. doi: https://doi.org/10.1080/13575279.2018.1521380.

Flewitt, R. et al. (2018). "I enjoyed it because ... you could do whatever you wanted and be creative": three principles for participatory research and pedagogy. International Journal of Research and Method in Education. Taylor \& Francis, 41(4), 372-386. doi: 10.1080/1743727X.2017.1405928.

Gallagher, M. (2008). "Power is not an evil": rethinking power in participatory methods. Children's Geographies. Routledge, 6(2), 137-150. https://doi: 10.1080/14733280801963045.

Gobierno de España (2015). Ley 26/2015, de 28 de julio, de modificación del sistema de protección a la infancia y a la adolescencia. España (en línea). https://www.boe.es/boe/dias/2015/07/29/pdfs/BOE-A-2015-8470.pdf.

Goodyer, A. (2014). Children's accounts of moving to a foster home. Child \& Family Social Work, 21(2), 188-197. https://doi: 10.1111/cfs.12128.

Hart, R. A. (1992). Children's Participation from Tokenism to Citizenship. Florence: Innocenti Research Centre.

Hernández, F. (2008). Educatio Siglo XXI, n. ${ }^{\circ} 26 \cdot 2008$, pp. 85-118 85 La investigación basada en las artes. Propuestas para repensar la investigación en educación. Educatio Siglo XX, 26, 85-118 (en línea).

http://revistas.um.es/educatio/article/viewFile/46641/44671.

Hernández, R. (2014). La investigación cualitativa a través de entrevistas: Su análisis mediante la teoría fundamentada. Cuestiones pedagógicas: Revista de ciencias de la educación, (23), 187-210.

Horgan, D. (2016). Child participatory research methods: attemts to go "deeper". Childhood, 1-15. https://doi: $10.1177 / 0907568216647787$.

Jackson, L., Deafenbaugh, L., Miller, E. (2018). Group metaphor map making: Application to integrated arts-based focus groups. Qualitative Social Work, 17(2), 305-322. https://doi: 10.1177/1473325016667475.

Lansdown, G. (2011). Every Child's Right to be Heard. London: Save the Children (en línea). http://www.unicef.org/files/Every_ Childs_Right_to_be_Heard.pdf.

Lansdown, G., O’Kane, C. (2014). A Toolkit for Monitoring and Evaluating Children's Participation. London: Save the Children.

Lara, E., Ballesteros, B. (2007). Métodos de investigación en educación social. Madrid: Universidad Nacional de Educación a Distancia.

Lay-Lisboa, S. et al. (2018). Protagonismo infantil en la escuela. Las relaciones pedagógicas en la construcción de ciudadanía. Sociedad e Infancias, 2, 147-170. https://doi: 10.5209/SOCI.59474.

Leitch, R., Mitchell, S. (2007). Caged birds and cloning machines: how student imagery "speaks" to us about cultures of schooling and student participation. Improving schools, 10(1), 53-71.

Lundy, L. (2007). Voice is not enough: Conceptualising Article 12 of the United Nations Convention on the Rights of the Child. British Education Research Journal, 33(6), 927-942. https://doi.org/10.1080/01411920701657033

Lundy, L., McEvoy, L. (2012). Children's rights and research processes: Assisting children to (in)formed views. Childhood, 19(1), 129-144.

https://doi.org/10.1177/0907568211409078

Mateos, A. et al. (2017). "They didn't tell me anything; they just sent me hom": children's participation in the return home. Child \& Family Social Work, 22(2), 871-880. https://doi: 10.1111/cfs.12307.

Mateos, A. et al. (2018). Good professional practices for promoting positive parenting and child participation in reunification processes. Child \& Family Social Work, 1-8. https://doi.org/10.1080/01411920701657033doi: 10.1111/cfs.12440.

Mauthner, M. (1997). Metodological aspects of collecting data from children: Lessons from three research projects. Children \& Society, 11, 16-28. https://doi.org/10.1111/j.1099-0860.1997.tb00003.x

Mitchell, M. B. et al. (2010). We care about care: advice by children in care for children in care, foster parents and child welfare workers about the transition into foster care. Child \& Family Social Work, 15(2), 176-185. doi: 10.1111/j.13652206.2009.00657.x.

Montserrat, C. (2014). The Child Protection System from the Perspective of Young People: Messages from 3 Studies. Social Sciences, 3(4), 687-704. https://doi: 10.3390/socsci3040687. 
Morgan, D. L. (2017). Conclusions: A Call for Further Innovations in Focus Groups. En R. S. Barbour, D. L. Morgan (eds), A new era in focus group research: Challenges, innovation and practice (pp. 411-420). London, UK: Palgrave McMillan. doi: 10.1057/978-1-137-58614-8_15.

ONU (1989). Convención sobre los Derechos del Niño y la Niña. Madrid: Ayuntamiento de San Fernando de Henares.

Palaiologou, I. (2014). "Do we hear what children want to say?" Ethical praxis when choosing research tools with children under five. Early Child Development and Care, 184(5), 689-705. https://doi: 10.1080/03004430.2013.809341.

Phoenix, C., Orr, N., Griffin, M. (2017). Bringing socio-narratology and visual methods to focus group research. En R. S. Barbour, D. L. Morgan (eds), A new era in focus group research: Challenges, innovation and practice (pp. 325-342). Palgrave McMillan. https://doi: 10.1057/978-1-137-58614-8_15.

Rodrigo, M. J. et al. (2013). Impact of the "Learning Together, Growing In Family" Programme on the Professionals and Attention to Families Services. Revista de cercetare si interventie sociala, 42, 145-165.

Rodríguez-Pinto, G., Albuquerque, L. (2018). “La magia que tenemos entre nosotros... niñas y niños”. Visibilidad, fuerza y afecto de la palabra infantil. Sociedad e Infancias, 2, 259-285. http://dx.doi.org/10.5209/SOCI.59509.

Schnoor, O. (2013). Early childhood studies as vocal studies: Examining the social practices of "giving voice to children's voices" in a crèche'. Childhood, 20(4), 458-471.

Santos, F. (2014). Cartas do Dixit (imagen en línea). <https://www.flickr.com/photos/farusantos/19608699574>, acceso el 3 de febrero de 2020.

Shier, H. (2001). Pathways to participation: Openings, opportunities and obligations. Children \& Society, 15(2), 107-117. https:// doi:10.1002/chi.617.

Shier, H. (2019). An analytical tool to help researchers develop partnerships with children and adolescents. En I. Berson, M. Berson, C. Gray (eds), Participatory Methodologies to Elevate Children's Voice and Agency (pp. 295-316). Charlotte, NC: Information Age Publishing.

Staines, J., Selwyn, J. (2020). "I wish someone would explain why I am in care": The impact of children and young people's lack of understanding of why they are in out-of-home care on their well-being and felt security. Child and Family Social Work, 1-10. https://doi: 10.1111/cfs.12721.

Susinos, T., Ceballos, N. (2012). Voz del alumnado y presencia participativa en la vida escolar. Apuntes para una cartografía de la voz del alumnado en la mejora educativa. Revista de Educacion, (359), 24-44. https://doi: 10.4438/1988-592X-RE-2012359-194.

Templeton, M. et al. (2019). Young people's sexual readiness: insights gained from comparing a researchers' and youth advisory group's interpretation. Culture, Health and Sexuality. Taylor \& Francis, 0(0), 1-14.https://doi: 10.1080/13691058.2019.1647555.

Tisdall, E. (2017). Conceptualising children and young people's participation: examining vulnerability, social accountability and co-production. The internacional journal of human rights, 21(1), 59-75.

https://doi: http://dx.doi.org/10.1080/13642987.2016.1248125.

Weber, S. J., Mitchell, C. (1996). Drawing ourselves into teaching: studying the images that shape and distort teacher education. Teaching and Teacher Education, 12(3), 303-313. https://doi.org/10.1016/0742-051X(95)00040-Q

Wetzel, R., Rodden, T., Benford, S. (2017). Developing Ideation Cards for Mixed Reality Game Design. Transactions of the Digital Games Research Association, 3(2), 275-211. https://doi: https://doi.org/10.26503/todigra.v3i2.73. 